\title{
Construcción de un marco de monitoreo para la salud universal
}

\author{
Ernesto Báscolo, ${ }^{1}$ Natalia Houghton ${ }^{1}$ y Amalia del Riego ${ }^{1}$
}

Forma de citar Báscolo E, Houghton N, del Riego A. Construcción de un marco de monitoreo para la salud universal. Rev Panam Salud Publica. 2018;42:e81. https://doi.org/10.26633/RPSP.2018.81

RESUMEN El objetivo del trabajo es construir un marco conceptual de monitoreo sobre los avances de politicas y acciones orientadas a alcanzar la salud universal. Se revisaron modelos conceptuales y propuestas metodológicas relacionados con el monitoreo del acceso y la cobertura universal de salud. Se realizó también una revisión de la literatura para seleccionar indicadores relevantes. Esta revisión fue complementada con un proceso de consulta con expertos en sistemas de salud de la Región de las Américas. Se desarrolló un marco integral para el monitoreo de politicas y acciones para el acceso y la cobertura universal de salud. El marco de monitoreo contiene cuatro componentes (acciones estratégicas, resultados inmediatos, resultados intermedios y resultados de impacto) e identifica un conjunto de opciones politicas para guiar la transformación de los sistemas de salud hacia el acceso y la cobertura universal de salud. Se eligieron 64 indicadores entre un total de 500 indicadores para la evaluación de los componentes del marco de monitoreo. El abordaje propuesto para la utilización del marco se basa en la medición de inequidades en las condiciones de acceso y cobertura, así como en la recolección de evidencia cualitativa sobre el grado de ejecución de políticas y acciones. El marco propuesto podría contribuir a fortalecer los procesos de transformación de los sistemas de salud para avanzar hacia el acceso y la cobertura universal de salud.

Palabras clave Atención de salud; políticas de salud; sistemas de salud; evaluación de la situación de salud.

En el año 2014, los Estados Miembros de la Organización Panamericana de la Salud (OPS) aprobaron la resolución CD53/5.R14 "Estrategia para el acceso universal a la salud y la cobertura universal de salud" (1). El objetivo fue definir un conjunto de intervenciones estratégicas para catalizar el fortalecimiento o transformación de los sistemas de

Organización Panamericana de la Salud, Washington, DC, Estados Unidos de América. Enviar correspondencia a Ernesto Báscolo, bascoloe@paho.org salud y lograr el acceso equitativo a la salud (1). La resolución explicita la equidad, la solidaridad y el derecho a la salud como valores fundamentales y reconoce la necesidad de un modelo de atención centrado en las personas y las comunidades como eje principal de la transformación de los sistemas de salud y un abordaje intersectorial que permita garantizar condiciones de acceso equitativo a dichos sistemas (1).

En esta resolución, se determina que el acceso universal a la salud y la cobertura universal de salud son dos fundamentos distintivos y complementarios de un sistema de salud equitativo. El acceso universal a la salud fue conceptualizado como "la ausencia de barreras de tipo geográfico, económico, sociocultural, de organización o de género que impiden que todas las personas utilicen servicios integrales de salud" (1). Por otra parte, la cobertura universal de salud se definió como "la capacidad del sistema de salud para responder a las necesidades de la población, lo cual incluye la disponibilidad

Este es un artículo de acceso abierto distribuido bajo los términos de la licencia Creative Commons Attribution-NonCommercial-NoDerivs 3.0 IGO, que permite su uso, distribución y reproducción en cualquier medio, siempre que el trabajo original se cite de la manera adecuada. No se permiten modificaciones a los artículos ni su uso comercial. Al reproducir un artículo no debe haber ningún indicio de que la OPS o el artículo avalan a una organización o un producto específico. El uso del logo de la OPS no está permitido. Esta leyenda debe conservarse, junto con la URL original del artículo. 
de infraestructura, recursos humanos, tecnologías de la salud (incluyendo medicamentos) y financiamiento" (1). El término "salud universal" fue posteriormente aprobado por la OPS como abreviatura aceptable para referirse al acceso universal a la salud y cobertura universal de salud (2).

Este enfoque renovado en la necesidad de transformar los sistemas de salud en la Región de las Américas resalta la necesidad de contar con un marco de referencia regional que permita medir los avances de las políticas orientadas al fortalecimiento de los sistemas de salud y el logro de la salud universal. Sin embargo, no existe un marco de monitoreo conceptual consensuado que ayude a los tomadores de decisiones a entender las implicaciones y efectos de las transformaciones del sistema de salud (3). La consistencia entre marcos conceptuales es importante porque facilita el trabajo colaborativo y el aprendizaje regional.

Si bien existen esfuerzos recientes para el monitoreo de la cobertura universal de salud (UHC, por sus siglas en inglés), en especial la adoptada en el marco de indicadores mundiales para los Objetivos de Desarrollo Sostenible (ODS) (4-6), estas propuestas se limitan a la medición de la cobertura de la población con servicios de salud y protección financiera (4-6). Aunque estas métricas ofrecen un entendimiento comprensivo de la calidad, pertinencia y asequibilidad financiera de los servicios de salud, por sí solas no ofrecen un panorama completo de las diferentes barreras de acceso a los servicios de salud y tampoco informan sobre el tipo de intervenciones que son necesarias para mejorar las condiciones de acceso $(7,8)$. En tanto, todavía no existe una propuesta regional para las Américas que considere en forma clara los mecanismos institucionales, políticos e intersectoriales propios de los procesos de trasformación del sistema de salud y que permita medir el impacto de esos esfuerzos en la salud universal.

Para abordar esta cuenta pendiente, la OPS inició, en octubre de 2014, un plan de trabajo para el desarrollo de un marco conceptual para el monitoreo de las políticas orientadas a alcanzar la salud universal. El propósito fue apoyar el análisis, la generación de evidencia y la toma de decisiones para la transformación o fortalecimiento de los sistemas de salud. En este artículo se describe el proceso y resultados de construcción de dicho marco de monitoreo.

\section{MATERIALES Y MÉTODOS}

Se utilizaron diferentes métodos, incluida una revisión de la literatura, consenso de expertos y entrevistas con informantes claves con base en abordajes empleados antes (9-11). Este proceso se efectuó entre abril de 2015 y noviembre de 2017 en las siguientes cuatro fases: conformación de un grupo de trabajo, consenso de expertos, pilotaje de la herramienta y recopilación y análisis de resultados (información adicional sobre la metodología empleada para la construcción del marco de monitoreo para la salud universal a disposición del lector interesado que la solicite al autor de correspondencia).

El grupo de trabajo fue constituido por tres personas con experticia en gobernanza de salud, liderazgo, política y planificación; monitoreo y evaluación; e investigación y análisis de sistemas de salud. Este grupo fue responsable de la selección del panel de expertos, formulación de criterios de consenso, revisión de literatura y desarrollo de propuesta del marco conceptual. Se revisaron diferentes modelos conceptuales y propuestas metodológicas relacionados con la salud universal, para la construcción del marco de monitoreo. Asimismo, se identificó un listado inicial de 500 indicadores a partir de un mapeo de bases de datos y marcos de monitoreo relacionados.

Para el consenso de expertos, se convocó a actores involucrados en la toma de decisiones habituales sobre los sistemas de salud, incluidos representantes de las oficinas técnicas de las autoridades nacionales de salud (ANS). Un total de 68 expertos y nueve países fueron invitados a fin de garantizar la diversidad de experiencias y representación regional: Brasil, Chile, Colombia, Costa Rica, Cuba, El Salvador, Panamá, Perú y Uruguay. Su ejecución requirió dos rondas de consulta, con procesamiento intermedio de opiniones e informe a los participantes. En ambas sesiones, los panelistas expertos recibieron una propuesta inicial del marco de monitoreo y criterios predefinidos para guiar el proceso de consulta.

La priorización de indicadores se basó en cinco criterios: 1) relevancia para avanzar hacia la salud universal; 2) validez (que mida lo que debería medir); 3) factibilidad (medible a través de encuestas de hogar, sistemas de información rutinarios y datos administrativos); 4) grado de disponibilidad entre países de la región y 5) fortalecimiento o complementariedad con otros marcos de monitoreo relacionados (ODS, UHC, Iniciativa de Atención Primaria en Salud).

La validez y factibilidad de los indicadores y metodología del marco de monitoreo fue estudiada a través de seis estudios piloto en Chile, Cuba, Jamaica, Panamá, Perú y Trinidad y Tabago. En estos países se convocó a oficiales del gobierno, la sociedad civil y otros actores del sistema de salud. Por último, se utilizaron entrevistas con informantes clave para analizar la opinión grupal a cada componente del marco de monitoreo. Para esto, el grupo de trabajo compiló y clasificó por área temática los comentarios ofrecidos durante las rondas de consulta de expertos y estudios piloto. El grupo de trabajo sostuvo entrevistas con 12 expertos en las áreas temáticas identificadas. El equipo de trabajo compiló y analizó por tema las entrevistas. Todas las diferencias se discutieron y resolvieron en el grupo.

En total, 314 expertos de 20 países participaron en una o más fases del proceso de construcción del marco de monitoreo y selección de indicadores para la salud universal.

\section{RESULTADOS}

Los expertos acordaron cuatro dimensiones de análisis: "acciones estratégicas", "resultados inmediatos", "resultados intermedios" y "resultados de impacto" (figura 1). Esta clasificación fue acordada conforme al mandato de la resolución CD53/5.R14 aprobada por los Estados Miembros de la OPS (1).

El orden y relación entre estas dimensiones también refleja la teoría y supuestos que respaldan la necesidad de formular y ejecutar de forma integrada políticas relacionadas a cada una de las acciones estratégicas para lograr tener un efecto sobre las condiciones de acceso y cobertura $(12,13)$.

Para cada dimensión, se acordó un total de 13 acciones estratégicas y 64 indicadores, incluidos 24 de resultado inmediato, 22 intermedios y 18 de impacto. Se acordaron también las definiciones, métodos de cálculo, desagregación y fuentes de información para cada indicador (información adicional sobre los indicadores cuantitativos para el monitoreo de 
FIGURA 1. Marco de monitoreo para el acceso y la cobertura universal de salud

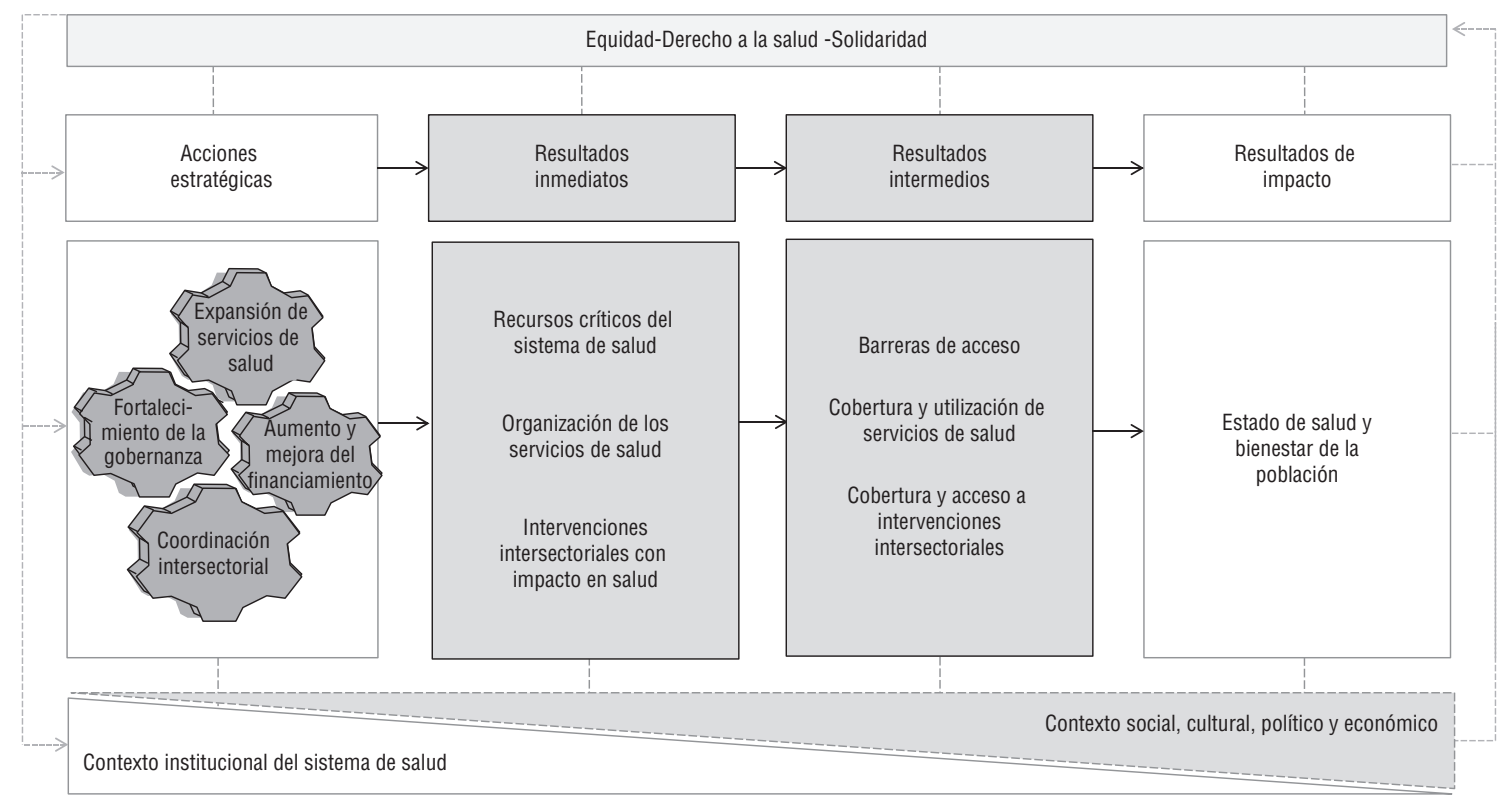

la salud universal a disposición del lector interesado que la solicite al autor de correspondencia).

\section{Componentes del marco de monitoreo para el acceso y la cobertura universal}

Acciones estratégicas. Las acciones estratégicas son las intervenciones mediante las cuales las autoridades de salud procuran fortalecer o transformar los sistemas de salud para alcanzar la salud universal.

Estas acciones estratégicas fueron agrupadas en cuatro líneas estratégicas conforme a la resolución CD53/5.R14 (1) (cuadro 1). En la primera línea, "ampliar el acceso equitativo a servicios de salud, integrales, de calidad, centrados en las personas y comunidades", el análisis aborda los avances en la provisión de servicios integrales, integrados y centrados en las necesidades de las personas y las comunidades, con especial énfasis en la capacidad resolutiva del primer nivel de atención y organización de los servicios en redes integradas. La segunda línea, "fortalecer la rectoría y la gobernanza", analiza la capacidad política y técnica de las ANS para conducir procesos de cambio de los sistemas de salud, como también de formular, regular y fiscalizar el cumplimiento de aquellos marcos normativos y regulatorios alineados a los valores de la salud universal.
En la tercera línea, "aumentar y mejorar el financiamiento con equidad y eficiencia y avanzar hacia la eliminación del pago directo que se convierte en barrera para el acceso en el momento de la prestación de servicios", se analiza el aumento y la optimización del financiamiento público de la salud, el fortalecimiento de la protección financiera, la minimización del pago directo de servicios y la mancomunación solidaria de fondos a fin de fortalecer el modelo de atención y asegurar el acceso universal. Por último, en la cuarta línea, "fortalecer la coordinación intersectorial para abordar los determinantes sociales de salud", se estudia la articulación e integración de los servicios de salud con diferentes sectores sociales, así como los mecanismos de regulación en la producción, comercialización y consumo de bienes y servicios con influencia sobre la salud de la población.

Resultados inmediatos. Dado que no resultaba práctico o factible medir indicadores para todas las acciones estratégicas, se seleccionaron 24 indicadores cuantitativos que dieran cuenta del efecto conjunto e integrado de las acciones estratégicas. Se agruparon estos indicadores en tres áreas: recursos críticos del sistema de salud (fuerza de trabajo en salud, financiamiento, medicamentos y tecnologías), organización de los servicios de salud, y acción intersectorial (cuadro 2).
Resultados intermedios. En total, se seleccionaron 22 indicadores cuantitativos agrupados en tres categorías para dar cuenta de la influencia de los resultados inmediatos sobre las condiciones de acceso: barreras de acceso, cobertura y utilización de servicios de salud y cobertura y acceso a intervenciones intersectoriales (cuadro 3).

Resultados de impacto. Los resultados de impacto incluyen 18 indicadores cuantitativos que se presentan como marcadores del estado de salud y bienestar de la población (cuadro 4).

\section{ABORDAJE PROPUESTO PARA EL USO DEL MARCO DE MONITOREO}

El monitoreo centrado en forma exclusiva en el seguimiento de tendencias, sin estar asociado a información contextual de los procesos de transformación del sistema de salud, no suele informar de manera apropiada sobre la efectividad de las políticas de gobierno (3). Por lo tanto, es necesario complementar el seguimiento con información cualitativa, recolectada mediante procesos sistemáticos, y que permita analizar las características y profundidad de los cambios introducidos en el sistema de salud (14).

Para responder a esta necesidad, los expertos acordaron una metodología para el uso del marco de monitoreo que vincule el análisis de equidad con el 
Línea estratégica 1. Ampliar el acceso equitativo a servicios de salud integrales, de calidad, centrados en las personas y las comunidades

AE 1.1. Aumentar la capacidad resolutiva del primer nivel de atención.

AE 1.2. Fortalecer la organización y la gestión de los servicios de salud a través de Redes Integradas de Servicios de Salud (RISS).

AE 1.3. Establecer mecanismos para incluir la participación del usuario de servicios, su familia y la comunidad en la toma de decisiones para mejorar la calidad de los cuidados y promover el autocuidado.

Línea estratégica 2. Fortalecer la rectoría y la gobernanza

AE 2.1. Desarrollar normas y estándares para mejorar la calidad en la prestación de servicios de salud.

AE 2.2. Asegurar la disponibilidad, distribución equitativa y calidad de los recursos humanos para la salud.

AE 2.3. Definir los procesos que mejoren la disponibilidad y regulación de los medicamentos y otras tecnologías sanitarias.

AE 2.4. Facilitar el empoderamiento de las personas y las comunidades y garantizar la representación de todos los grupos de la población en el proceso de formulación de las políticas para fortalecer la coordinación entre salud y comunidad.

AE 2.5. Fortalecer los sistemas de información con datos desagregados a niveles nacionales y subnacionales disponibles para identificar las necesidades de salud, desigualdades en salud y barreras de acceso.

AE 2.6. Priorizar la investigación sobre salud universal en la agenda nacional de investigación.

Línea estratégica 3. Aumentar y mejorar el financiamiento, con equidad y eficiencia, y avanzar hacia la eliminación del pago directo que se convierte en barrera para el acceso en el momento de la prestación de servicios

AE 3.1. Utilizar la reglamentación fiscal como instrumento para promover la movilización y la asignación de recursos financieros para la salud.

AE 3.2. Avanzar hacia la complementariedad de los recursos para la salud entre diversas fuentes.

Línea estratégica 4. Fortalecer la coordinación multisectorial para abordar los determinantes sociales de la salud que garanticen la sostenibilidad de la cobertura universal

AE 4.1. Establecer o fortalecer mecanismos de coordinación intersectorial.

AE 4.2. Establecer o fortalecer la capacidad de la autoridad nacional de salud para implementar con éxito políticas públicas intersectoriales.

AE; acción estratégica.

\section{CUADRO 2. Indicadores de resultado inmediato en salud universal}

Recursos críticos del sistema de salud

- Densidad y distribución de trabajadores de salud.

- Porcentaje de equipos de profesionales de salud o especialistas médicos que reciben remuneración con pago por desempeño para incrementar el acceso y calidad de servicios de salud en la población a cargo.

- Gasto público y privado per cápita (en dólares estadounidenses) en productos farmacéuticos.

- Número de unidades de teleterapia de alta energía (cobalto-60 y aceleradores lineales) por millón de habitantes.

- Tasa de donación de sangre por 1000 personas.

- Cobertura poblacional por esquemas de financiamiento de la salud.

- Proporción de gasto público destinado a la salud en relación al producto interno bruto (PIB).

- Porcentaje del gasto público en el primer nivel de atención con respecto al gasto público total en salud.

Organización de los servicios de salud

- Porcentaje de hospitales que tienen financiamiento del presupuesto histórico y pago por actoa.

- Porcentaje de hospitales que tienen financiamiento prospectivo basado en productos sanitarios.

- Porcentaje de hospitalizaciones prevenibles por condiciones sensibles al cuidado ambulatorio.

- Prevalencia de usuarios con infecciones asociadas a la atención en salud.

- Satisfacción de los usuarios con los servicios de salud.

- Porcentaje de establecimientos de primer nivel de atención con población a cargo en base territorial.

- Porcentaje de la población nacional que tiene cobertura por Redes Integradas por Servicios de Salud (RISS).

Intervenciones intersectoriales con impacto en salud

- Proporción de la población con acceso a servicios de agua potable.

- Proporción de la población que utiliza una instalación de saneamiento mejorada. aPresupuesto histórico: sistemas presupuestarios que se reproducen basados en la formulación y ejecución de presupuestos de años anteriores.

análisis de políticas. Para esto, se contempla la desagregación de indicadores según diferentes variables socioeconómicas, así como la colección de información cualitativa que informe sobre el grado de ejecución de políticas clave de transformación del sistema de salud. Para el análisis de datos cuantitativos, se espera que los ministerios de salud seleccionen, dentro del listado de 60 indicadores, aquellos que son más relevantes al propio contexto nacional, teniendo en cuenta la disponibilidad de información y las prioridades del sistema de salud, incluido el perfil epidemiológico y las necesidades de políticas de transformación del sistema de salud.

El objetivo del abordaje cualitativo es complementar el análisis de datos cuantitativos a través de información contextual que dé cuenta de las características y profundidad de los cambios introducidos por las políticas del sistema de salud, e identificar opciones de políticas para abordar las brechas y desafíos encontrados (15-18). Para esto, se desarrolló un cuestionario para la colección de información en donde cada acción estratégica es analizada en función de criterios organizacionales que permitan interpretar la capacidad de los servicios de salud para dar respuesta a las necesidades de salud de la población, aspectos institucionales con influencia sobre los marcos regulatorios y los mecanismos de asignación de recursos (financieros, tecnológicos y humanos) para fortalecer el sistema de salud, criterios políticos, reflejados por las acciones producidas por la ANS y otros actores que hacen posibles los cambios institucionales y los mecanismos de asignación de recursos, y el reconocimiento de aquellas intervenciones intersectoriales que facilitan estos procesos (información adicional sobre el análisis de las acciones estratégicas a disposición del lector interesado que la solicite al autor de correspondencia). 


\section{CUADRO 3. Indicadores de resultados intermedios}

Barreras de acceso

- Porcentaje de la población reportando barreras de acceso a la salud (culturales, institucionales [aceptabilidad, conveniencia, disponibilidad, tiempo de espera], económicas, geográficas).

- Proporción del gasto de bolsillo en salud con respecto al gasto total en salud.

- Porcentaje de la población que experimenta gastos de bolsillo en salud catastrófico.

- Porcentaje de hogares que experimentan gastos de bolsillo que causa empobrecimiento.

Cobertura y utilización de servicios de salud

- Cobertura con tres dosis de la vacuna DPT en el primer año de vida.

- Proporción de mujeres de 30 a 49 años de edad que declaran haberse sometido a detección del cáncer cervicouterino.

- Necesidades insatisfechas con respecto a la planificación familiar.

- Proporción de población gestante atendida por personal calificado durante el embarazo.

- Porcentaje de partos atendidos por personal de salud calificado.

- Porcentaje de visitas de salud preventivas en un año.

- Acceso a programas de salud de la comunidad para adultos mayores.

- Cobertura de atención de las personas con discapacidad.

- Porcentaje de hipertensión controlada a nivel de población en personas de 18 años o más.

- Porcentaje de diabetes controlada a nivel de población en personas de 18 años o más.

- Tratamiento en establecimientos ambulatorios de salud mental.

- Porcentaje de cobertura con tratamiento antirretroviral.

- Cobertura con tratamiento profiláctico del VIH para la prevención de la transmisión maternoinfantil.

- Porcentaje de cobertura de las embarazadas con el tratamiento para la sífilis.

- Porcentaje de pacientes con tuberculosis tratados con éxito.

- Tiempo medio entre el inicio de los síntomas y el inicio del tratamientode la malaria.

- Porcentaje de casos diagnosticados y tratados de leishmaniasis.

- Porcentaje de cobertura con tratamiento de hepatitis viral.

Cobertura y acceso a intervenciones intersectoriales

- Uso de combustibles sólidos.

- Consumo de alcohol por habitante en los mayores de 15 años.

- Consumo de tabaco en adolescentes.

- Consumo de tabaco en adultos.

- Actividad física insuficiente en los adolescentes.

- Actividad física insuficiente en adultos.

- Lactancia materna en menores de seis meses.

DPT, vacuna triple bacteriana (tétanos, difteria y tos convulsa); VIH, virus de la inmunodeficiencia humana.

\section{CUADRO 4. Indicadores de resultados de impacto}

- Esperanza de vida sana

- Tasa de mortalidad infantil

- Razón de mortalidad materna

- Tasa de mortalidad por causas evitables mediante la atención de salud

- Muerte prematura por enfermedades no transmisibles y factores de riesgo (enfermedades cardiovasculares; tumores malignos, diabetes mellitus y enfermedades respiratorias crónicas)

- Tasa de mortalidad por VIH/sida

- Tasa de mortalidad por tuberculosis

- Transmisión maternoinfantil de la infección por el VIH y la sífilis congénita

- Tasa de homicidios en jóvenes de 15 a 24 años de edad

- Tasa de muertes por suicidio

- Tasa de mortalidad debida a traumatismos causados por el tránsito en jóvenes de 15 a 24 años de edad

- Sobrepeso y obesidad

- Prevalencia estandarizada de glucemia elevada y diabetes en personas de 18 años o más

- Proporción de adultos mayores con discapacidad AVD

- Bajo peso al nacer $(<2500 \mathrm{~g})$

- Prevalencia de desnutrición crónica en niños menores de cinco años

- Tasa específica de fecundidad en mujeres de 15 a 19 años de edad

- Prevalencia de violencia de pareja

AVD, actividades de la vida diaria.

\section{DISCUSIÓN}

En este artículo se describe el proceso y resultados de construcción de un marco conceptual para el monitoreo de la salud universal. Este fue desarrollado a partir de un proceso de consulta con expertos y validado en reuniones regionales y estudios piloto. Es importante contar con un marco consensuado que acompañe los procesos de transformación de los sistemas de salud para guiar las acciones dirigidas a avanzar hacia la salud universal en la Región de las Américas.

Lograr la salud universal implica la formulación de políticas y acciones innovadoras que aceleren la transformación de los sistemas de salud. Desde esta perspectiva, el marco de monitoreo debería facilitar estos procesos de cambio. Su construcción consensuada permitió generar un instrumento donde los actores clave estuvieron de acuerdo con la relevancia, validez y factibilidad de las métricas y metodología elegidas. La aceptabilidad de la herramienta es un elemento clave para facilitar procesos de cambio (19).

Asimismo, la inclusión de varias dimensiones de análisis (acciones estratégicas, resultados inmediatos, resultados intermedios y resultados de impacto) hace posible un abordaje mixto, con métodos cualitativos y cuantitativos, para explicar mejor el efecto de las intervenciones políticas en las condiciones de acceso y cobertura. Este abordaje metodológico también debería guiar el diseño y desarrollo de procesos de evaluación más profundos sobre el efecto de las políticas de transformación del sistema de salud, incluidos los procesos, los factores contextuales y la causalidad.

La construcción del marco de monitoreo también incluyó una revisión de propuestas metodológicas existentes. La incorporación de muchas de las recomendaciones y métricas presentes en dichos instrumentos fue fundamental para lograr armonizar los diferentes esfuerzos globales y ofrecer un léxico común para la comunicación entre actores. Sin embargo, los instrumentos disponibles a nivel global fueron considerados insuficientes para el monitoreo de políticas en el contexto de la Región de las Américas.

En época reciente, Hogan y colaboradores (6) desarrollaron un índice de cobertura de servicios esenciales de salud para la medición de UHC. Al igual que 
en otros esfuerzos recientes $(4,5,19-21)$, el abordaje empleado se basa en la agrupación de varias métricas de cobertura de servicios de salud, disponibilidad de recursos o estado de salud para crear una medida sintética de la UHC. Si bien este tipo de abordaje tiene grandes fortalezas, sobre todo por su simplicidad metodológica, relevancia y uso de datos disponibles en las encuestas de hogar, el análisis de los datos presenta grandes desafíos para el monitoreo efectivo de políticas para la salud universal.

En primer lugar, al combinar diferentes indicadores en una sola métrica sintética, es difícil atribuir cambios en la cobertura de servicios a diferencias concretas en el desempeño de políticas, ya que los cambios observados pueden ser más bien el reflejo de diferencias en las encuestas utilizadas, los distintos tiempos de recolección, la disponibilidad de datos o todos ellos. Más aun, el foco en el ranking entre países, sumado a la ausencia de información contextual sobre los procesos de trasformación del sistema de salud, dificultan la interpretación de los resultados del índice. Por ejemplo, los puntajes de Brasil, Costa Rica y Cuba, países ampliamente reconocidos por sus significativos avances en el acceso y cobertura universal de salud, son iguales que los de El Salvador, Ecuador y Perú, respectivamente (6). Resulta entonces difícil entender cómo estos países pueden tener resultados similares en términos de acceso y cobertura universal.

Para apoyar el desarrollo del abordaje metodológico propuesto en este marco de monitoreo, se consideraron instrumentos de tipo cualitativo usados para la producción y análisis del desempeño de los sistemas de salud. Ejemplos de tales instrumentos incluyen el marco para el monitoreo de los Elementos Esenciales de los Sistemas de Salud de la OMS (22), los perfiles de sistemas de salud del Observatorio Europeo de Políticas y Sistemas de Salud (23) y el compendio de indicadores en salud de la Organización de Cooperación y Desarrollo Económicos (OCDE) (24). Una de las principales fortalezas observadas en estos instrumentos es la inclusión de marcos conceptuales en los que se identifican diferentes dimensiones que guían el análisis e interpretación de los resultados en términos de desempeño de los sistemas de salud $(23,24)$. Sin embargo, cada dimensión tiende a analizarse de forma independiente, sin definir relaciones de causalidad o presentar un análisis integral de políticas.

Otros instrumentos, incluida la Metodología de Evaluación del Desempeño de los Sistemas de Salud (PROADESS) de la Fundación Oswaldo Cruz (25), los perfiles de sistemas de salud del Instituto Suramericano de Gobierno en Salud (ISAGS) (26) y la plataforma virtual del Observatorio Iberoamericano de Políticas y Sistemas de Salud (OIAPSS) (27), cuentan con matrices de análisis en las que se propone un abordaje integral de dimensiones e indicadores de desempeño del sistema de salud. Estas dimensiones organizan el análisis de los resultados de los sistemas de salud y contemplan el análisis de la rectoría del sistema de salud como eje estructurador de los sistemas. Si bien estos abordajes tienen similitudes con el marco de monitoreo propuesto en este manuscrito, no cuentan con la identificación de opciones de políticas explicitas que guíen el análisis de los procesos de transformación del sistema de salud.

Aunque existen otros esfuerzos para desarrollar instrumentos de monitoreo y evaluación de la Atención Primaria en Salud (APS) (28-30), incluido el Primary Health Care Performance Initiative (PHCPI) (28), el enfoque de estos instrumentos se limita a la APS y provisión de servicios y no al análisis integral de los procesos de trasformación del sistema en su conjunto. Con base en la revisión de los instrumentos realizados, se consideró importante agregar un análisis integral de los cambios en los mecanismos institucionales, políticos e intersectoriales, propios de la dinámica y naturaleza de los procesos de transformación de los sistemas de salud $(12,13)$. En efecto, la integralidad de estos procesos es fundamental para robustecer la ampliación de la cobertura de los recursos críticos del sistema, garantizar el acceso a los servicios de salud, y, luego, generar cambios en el impacto en los resultados en salud (12).

El desarrollo y validación del marco de monitoreo no estuvo exento de desafíos. Una de las recomendaciones surgidas durante el proceso de consulta con expertos se basa en la dificultad de analizar el listado completo de indicadores y acciones estratégicas. En efecto, el análisis de los dominios incluidos en el marco de monitoreo puede ser relativamente complejo para países grandes donde la responsabilidad de la ejecución de políticas recae sobre diferentes autoridades a nivel federal, estatal y local. Esto representa un desafío a la hora de interpretar el análisis de políticas a niveles subnacionales. Por esta razón, el desarrollo de un instrumento robusto permite que las autoridades de salud puedan seleccionar aquellas dimensiones e indicadores más relevantes para su contexto nacional.

Otros desafíos radican en la poca disponibilidad de datos desagregados por variables socioeconómicas para el monitoreo de la equidad. Por otra parte, el listado de indicadores incluidos no es exhaustivo y se reconoce que existen múltiples indicadores del estado de salud. Sin embargo, resulta más práctico elegir un conjunto de indicadores trazadores que pueda ser adaptado por los países en función de sus necesidades en salud. Para esta adaptación, los expertos recomendaron que cada país priorice aquellos indicadores y acciones estratégicas que sean más relevantes y factibles para su contexto particular.

Si bien la existencia de un marco consensuado para la Región de las Américas facilita el aprendizaje regional sobre las implicaciones y efectos de las transformaciones del sistema de salud, el desarrollo del marco no fue concebido para hacer comparaciones entre países. La intención principal es apoyar el seguimiento de las metas nacionales de cada país y apoyar la identificación de áreas que necesitan mayor atención y la introducción de medidas correctivas.

Por último, el desarrollo del marco de monitoreo presentado es solo un insumo inicial. La producción, análisis y uso de información sobre la salud universal requiere fortalecer sistemas robustos de monitoreo a nivel nacional. El fortalecimiento de la gobernanza de los sistemas de monitoreo, además de una mayor inversión, son esenciales para cubrir las necesidades de análisis y seguimiento de las políticas para la salud universal, así como las de otros programas y actividades del sistema de salud. Se requiere fortalecer los sistemas de información nacionales que integren datos de encuestas de hogares e instituciones de salud y que permitan un adecuado flujo de datos. Estos y otros elementos relacionados con la construcción de sistemas nacionales de monitoreo requieren ser abordados en futuros estudios.

Agradecimientos. Los autores agradecen a todos los participantes del taller de expertos realizado en Santiago de Chile en abril de 2015 y las numerosas consultas 
nacionales realizadas durante el período de junio del 2015 a noviembre del 2016. En particular, agradecen la importante contribución de la Universidad del Desarrollo de Chile, del Ministerio de Salud de Chile, del Ministerio de Salud de Cuba, del Ministerio de Salud de Jamaica, del Ministerio de Salud de Panamá y del Ministerio

1. Organización Panamericana de la Salud (OPS). Estrategia para el acceso universal a la salud y la cobertura universal de salud. 53. ${ }^{\circ}$ Consejo Directivo de la OPS, 66. ${ }^{a}$ Sesión del Comité Regional de la OMS para las Américas. (Resolución CD53/5. R2). Washington, D.C.: OPS; 2014.

2. Organización Panamericana de la Salud. Dos palabras que recordar. Disponible en: https: / /intra.paho.org/Pages/abreviando.aspx Acceso el 28 de marzo de 2018.

3. Obermann K, Chanturidze T, Richardson E, Tanirbergenov S, Shoranov M, Nurgozhaev A. Data for development in health: a case study and monitoring framework from Kazakhstan. BMJ Global Health. 2016;1(1): e000003. doi:10.1136/bmjgh-2015-000003

4. Organización Mundial de la Salud y Banco Mundial (OMS/BM). Monitoreo del progreso hacia la cobertura universal de salud a nivel nacional y global. Ginebra: OMS/ BM; 2014. Disponible en: http://apps. who.int/iris/bitstream/10665/112827/1/ WHO_HIS_HIA_14.1_spa.pdf?ua $=1$ Acceso el 12 de enero de 2018.

5. Asamblea General de la Naciones Unidas. Marco de indicadores mundiales para los Objetivos de Desarrollo Sostenible y metas de la Agenda 2030 para el Desarrollo Sostenible (Resolución A/RES/71/313). Nueva York: Naciones Unidas; 2017. Disponible en: https://unstats.un.org/ sdgs/indicators/Global\%20Indicator $\% 20$ Framework_A.RES.71.313\%20Annex. Spanish.pdf

6. Hogan D, Stevens GA, Reza A, Boerma T. Monitoring universal health coverage within the Sustainable Development Goals: development and baseline data for an index of essential health services. Lancet Global Health. 2018;6(2):e152-e168. doi: http://dx.doi.org/10.1016/S2214-109X(17) 30472-2

7. Thorpe JM, Thorpe CT, Kennelty KA, Pandhi N. Patterns of perceived barriers to medical care in older adults: a latent class analysis. BMC Health Services Research. 2011;11:181. doi: 10.1186/1472-6963-11-181.

8. Travassos C, Martins M. Uma revisão sobre os conceitos de acesso e utilização de serviços de saúde. Cad Saude Publica. 2004;20(S2):S190-8.

9. Bryce J, Requejo JH, Moulton LH, Ram M, Black RE. A common evaluation framework for the African Health Initiative. BMC Health Services Research. 2013;13(S2):S10. doi:10.1186/1472-6963-13-S2-S10

10. Jahanmehr N, Rashidian A, Khosravi A, Farzadfar F, Shariati M, Majdzadeh R, et al. A conceptual framework for evaluation of de Salud de Perú en la organización y aportes ofrecidos durante varias de las consultas mencionadas anteriormente. Asimismo, agradecen a Gisele Almeida, Hernán Montenegro, Ricardo Fábrega, Camilo Cid, Cristian Morales y Ximena Aguilera por comentar en detalle las primeras versiones de este documento.

\section{REFERENCIAS}

public health and primary care system performance in Iran. Global Journal of Health Science. 2015;7(4):341-57. doi:10.5539/gjhs. v7n4p341

11. Moran AC, Jolivet RR, Chou D, Dalglish SL, Hill K, Ramsey K, et al. A common monitoring framework for ending preventable maternal mortality, 2015-2030: phase I of a multi-step process. BMC Pregnancy and Childbirth. 2016;16(1):250. doi:10.1186/ s12884-016-1035-4

12. Organización Panamericana de la Salud (OPS). Salud en las Américas 2017: Rectoría y gobernanza hacia la salud universal. Washington D.C.: OPS; 2017. Disponible en: http://www.paho.org/salud-en-lasamericas $-2017 /$ ?post_type $=$ post_t_es\& $\mathrm{p}=309 \&$ lang=es Acceso el 29 de marzo de 2018.

13. Organización Panamericana de la Salud (OPS). Salud en las Américas 2017: Transformaciones de los sistemas de salud hacia la salud universal. Washington D.C.: OPS; 2017. Disponible en: http://www.paho. org/salud-en-las-americas-2017/?post type $=$ post_t_es\&p $=305 \& l a n g=e s$ Acceso el 29 de marzo de 2018.

14. World Health Organization (WHO). Monitoring and evaluation of health systems strengthening: an operational framework. Geneva: WHO; 2009. Disponible en: http:/ / www.who.int/healthinfo/HSS MandE_framework_Nov_2009.pdf Consultado el 5 de abril del 2018.

15. Adler ES, Clark R. How it's done: an invitation to social research. Belmont, CA: Wadsworth; 2008.

16. Bernard HR. Social research methods: qualitative and quantitative approaches. Thousand Oaks, CA: Sage; 2000.

17. Miles M, Huberman M. Qualitative data analysis. Thousand Oaks, CA: Sage; 1994.

18. Campbell SM, Braspenning J, Hutchinson A, Marshall MN. Research methods used in developing and applying quality indicators in primary care. BMJ. 2003;326(7393): 816-19.

19. The PLOS Medicine Editors. Monitoring universal health coverage collection: managing expectations. PLOS Medicine. 2014; 11(9):e1001732.

20. Lim SS, Allen K, Bhutta ZA, Dandina L, Forouzanfar $\mathrm{MH}$, Fullman $\mathrm{N}$, et al. Measuring the health-related Sustainable Development Goals in 188 countries: a baseline analysis from the Global Burden of Disease Study 2015. Lancet. 2016;388(10053): 1813-50.

21. Fullman N, Barber RM, Abajobir AA, Abate $\mathrm{KH}$, Abbafati C, Abbas KM, et al. Measuring
Conflicto de intereses. Ninguno declarado por los autores.

Declaración. Las opiniones expresadas en este manuscrito son responsabilidad del autor y no reflejan necesariamente los criterios ni la política de la RPSP/PAJPH y/o de la OPS. progress and projecting attainment on the basis of past trends of the health-related Sustainable Development Goals in 188 countries: an analysis from the Global Burden of Disease Study 2016. Lancet. 2017;390(10100):1423-59.

22. World Health Organization (WHO). Monitoring the building blocks of health systems: a handbook of indicators and their measurement strategies. Geneve: WHO 2010. Disponible en: http: / /www.who.int/ healthinfo/systems/WHO_MBHSS_2010_ full_web.pdf Acceso el 12 de enero de 2018.

23. World Health Organization. The Health Systems in Transition (HiT): Template for authors. Geneva: WHO/European Observatory on Health Systems and Policies; 2010.

24. Organización de Cooperación y Desarrollo Económicos (OCDE). Health at a glance 2015: OECD indicators. París: OCDE; 2015. Disponible en: http:/ / apps.who.int/medicinedocs/documents/s22177en/s22177en. pdf Acceso el 12 de enero del 2018.

25. Viacava F, Almeida C, Caetano R, Fausto M, Macinko J, Martins M et al. Uma metodologia de avaliação do desempenho do sistema de saúde brasileiro. Cienc. saude coletiva. 2004;9(3):711-24.

26. Instituto Suramericano de Gobierno en Salud (ISAGS). Health Systems in South America: challenges to the universality, integrality and equity. Quito: ISAGS; 2012.

27. Observatório Ibero-Americano de Políticas e Sistemas de Saúde: Matriz analítica. Disponible en: http://www.oiapss.org/?page_id=2581 Acceso el 12 de enero de 2018.

28. Primary Health Care Performance Initiative (PHCPI). Indicadores. Disponible en: https://www.phcperformanceinitiative. org/about-us/about-phcpi Acceso el 12 de enero de 2018.

29. Haggerty JL, Yavich N, Báscolo EP. Grupo de Consenso sobre un Marco de Evaluación de la Atención Primaria en América Latina. Un marco de evaluación de la atención primaria de salud en América Latina. Rev Panam Salud Publica. 2009;26(5):377-84.

30. Yavich N, Báscolo EP, Haggerty J. Construyendo un marco de evaluación de la atención primaria de la salud para Latinoamérica. Salud Publica Méx. 2010;52(1): 39-45.

Manuscrito recibido el 18 de enero de 2018. Aceptado para publicación, tras revisión, el 04 de abril de 2018. 
ABSTRACT The objective of the study is to construct a conceptual framework for monitoring progress on policies and actions aimed at reaching universal health.

\section{Construction of a monitoring framework for universal health}

Keywords
Conceptual models and proposed methodologies for monitoring universal health coverage and access were reviewed. A literature review was also done to select relevant indicators. This review was supplemented with a process of consultation with health systems experts from the Region of the Americas.

A comprehensive framework was developed for monitoring policies and actions for universal health coverage and access. This monitoring framework contains four components (strategic actions, outputs, outcomes, and impacts) and identifies a set of policy options to guide the transformation of health systems toward universal access to health and universal health coverage. Sixty-four out of 500 indicators were chosen for evaluation of the monitoring framework components. The proposed approach for use of the framework is based on measuring inequities in access and coverage, and on collecting qualitative evidence on the degree to which policies and actions have been implemented.

The proposed framework could help strengthen health system in processes of transformation toward universal access to health and universal health coverage.

Health care; health policy; health system; health situation assessment.

RESUMO Construir um quadro conceitual de monitoramento do progresso de políticas e ações voltadas à saúde universal.

Foram examinados modelos conceituais e propostas metodológicas de monitoramento do acesso universal à saúde e cobertura universal de saúde. Foi realizada

\section{Construção de um quadro de monitoramento para saúde universal} também uma revisão da literatura para selecionar os indicadores relevantes, complementada com um processo de consulta com especialistas em sistemas de saúde da Região das Américas.

Foi elaborado um quadro completo para o monitoramento de políticas e ações para o acesso universal à saúde e a cobertura universal de saúde. O quadro de monitoramento contém quatro componentes (ações estratégicas, resultados imediatos, resultados intermediários e resultados de impacto) e expõe uma série de opções políticas para direcionar a transformação dos sistemas de saúde para o acesso universal à saúde e cobertura universal de saúde. Foram selecionados 64 de um total de 500 indicadores para avaliar os componentes do quadro de monitoramento. A abordagem proposta para a aplicação do quadro se baseia na mensuração das iniquidades das condições de acesso e cobertura e na coleta de evidências qualitativas do nível de implementação de políticas e ações.

O quadro proposto pode contribuir para consolidar os processos de transformação dos sistemas de saúde rumo ao acesso universal à saúde e cobertura universal de saúde.

Palavras-chave 